\title{
GENERALIZED THERMOELASTIC INTERACTIONS DUE TO AN INCLINED LOAD AT A TWO-TEMPERATURE HALF-SPACE
}

\author{
Ahmed E. Abouelregal \\ Department of Mathematics, Faculty of Science, Mansoura University, Mansoura 35516, Egypt and \\ Department of Mathematics, College of Science and Arts, Aljouf University, Al-Qurayat, Saudi Arabia \\ Ashraf M. Zenkour \\ Department of Mathematics, Faculty of Science, King Abdulaziz University, Jeddah, Saudi Arabia and \\ Department of Mathematics, Faculty of Science, Kafrelsheikh University, Kafr El-Sheikh, Egypt \\ e-mail: zenkour@sci.kfs.edu.eg
}

\begin{abstract}
The article presents a two-temperature theory to study the thermally insulated stress-free surface of a thermoelastic solid half-space due to an inclined load. The inclined load is a linear combination of a normal load and a tangential load. The normal mode analysis has been employed to solve the present problem. Variations of conductive and thermodynamic temperatures, displacements, and stresses distributions with the horizontal distance have been presented graphically. Some comparisons have been made to estimate the effects due to the two-temperature parameter and the inclination angle on the field quantities. Results of earlier works have been deduced from the present investigation as special cases.
\end{abstract}

Keywords: thermoelasticity, conductive and thermodynamic temperatures, half-space, inclined load

\section{Introduction}

Classical thermoelasticity theory is based on Fourier's law of heat conduction, which, when combined with other fundamental field equations, leads to coupled hyperbolic-parabolic governing equations. These equations imply that thermal effects are to be felt instantaneously far away from the external thermo-mechanical load. Therefore, this theory admits infinite speeds of propagation of thermoelastic disturbances. This paradox becomes especially evident in problems involving very short time intervals or high rates of heat flux.

The heat equations for both the classical uncoupled theory and the coupled one by Biot (1956) of the diffusion type predict infinite speeds of propagation for heat waves contrary to physical observations. The classical uncoupled theory states that the elastic changes have no effect on temperature. So, Biot (1956) formulated his theory to eliminate this paradox. At present, there are several theories of hyperbolic thermoelasticity (Lord and Shulman, 1967; Green and Lindsay, 1972) with one and two relaxation times. Both of these theories ensure finite speeds of propagation for the heat wave. Green and Naghdi (1993) formulated another generalized thermoelasticity theory without energy dissipation. It included isothermal displacement gradients among its independent constitutive variables. Recently, Zenkour (2015) presented a unified theory that included different generalized and coupled thermoelasticity theories.

Sherief and Hamza (1996) solved a 2-D problem in spherical regions. Sherief and El-Maghraby (2003, 2005) solved two problems including cracks in an infinite thermoelastic solid. A 2-D problem for a half-space and for a thick plate under the action of body forces was solved by El-Maghraby (2008, 2009). Allam et al. (2009) studied a 2-D problem of electromagnetothermoelasticity for a homogeneous isotropic perfectly conducting thick plate. Abouelregal and 
Zenkour (2013) presented the effect of fractional thermoelasticity in a rotating fibre-reinforced thermoelastic medium. Zenkour and Abouelregal (2015) investigated a thermoelastic problem of an axially moving microbeam subjected to an external transverse excitation.

The present article is concerned with investigations related to analytical expressions for displacement, stress, and temperature distributions on the free surface of a thermoelastic medium subjected to an inclined load. The governing equations are taken in the two-temperature generalized thermoelasticity model (for the two-temperature model, one can refer to (Chen and Gurtin, 1968; Chen et al., 1969; Boley, 1956; Abbas and Zenkour, 2014; Zenkour and Abouelregal, 2014a,b). Some comparisons are graphically shown to estimate the effect of the inclination angle and the two-temperature parameters on all the studied fields.

\section{Basic equations}

The basic equations of motion for a linear, homogeneous and isotropic thermoelastic material in the context of generalized thermoelasticity with two temperatures in the absence of the body force take the following forms

$$
\mu \nabla^{2} \mathbf{u}+(\lambda+\mu) \nabla \operatorname{div} \mathbf{u}-\gamma \nabla \theta=\rho \ddot{\mathbf{u}}
$$

where $\mathbf{u}$ denotes the displacement vector, $\theta=T-T_{0}$ denotes the thermodynamical temperature, $T_{0}$ is the reference temperature, $\lambda$ and $\mu$ are Lamé's constants, $\gamma=(3 \lambda+2 \mu) \alpha_{t}, \alpha_{t}$ is the coefficient of thermal expansion and $\rho$ is the mass density. Note that the direct vector/tensor notation is employed; also, an over dot denotes partial derivative with respect to the time variable $t$.

The stress tensor $\boldsymbol{\sigma}$ associated with $\mathbf{u}$ and $\theta$ is given by the constitutive equation

$$
\boldsymbol{\sigma}=\lambda(\operatorname{div} \mathbf{u}) \mathbf{I}+\mu\left[\nabla \mathbf{u}+(\nabla \mathbf{u})^{\mathrm{T}}\right]-\gamma \theta \mathbf{I}
$$

where $\mathbf{I}$ is the identity tensor and the suffix $T$ is the transpose of the given vector.

The strain-displacement relations are

$$
e_{i j}=\frac{1}{2}\left(u_{i, j}+u_{j, i}\right)
$$

where $e_{i j}$ is the strain tensor.

The equation of entropy (energy equation) is

$$
\rho T_{0} \frac{\partial \eta}{\partial t}=-q_{i, i}+Q
$$

where $\eta$ is the entropy per unit volume measured from the entropy of the reference state, $q_{i}$ are the components of the heat flow vector, and $Q$ is the applied heat flux.

The entropy-strain-temperature relation is

$$
\rho \eta=\gamma e_{k k}+\frac{\rho C_{E}}{T_{0}} \theta
$$

where $C_{E}$ is the specific heat at constant strain and $e_{k k}=\operatorname{div} \mathbf{u}=e$ is the cubical dilatation.

The modified Fourier law is

$$
q_{i}+\tau_{0} \frac{\partial q_{i}}{\partial t}=-K \varphi_{, i}
$$


where $\tau_{0}$ is the thermal relaxation time which will ensure that the heat conduction equation will predict finite speeds of heat propagation, $K$ is the thermal conductivity, and $\varphi$ is the conductive temperature measured from the temperature $\varphi_{0}\left(\varphi_{0}=T_{0}\right)$, which satisfies the relation

$$
\theta=\varphi-a \varphi_{, i i}
$$

where $a$ is the two-temperature parameter (temperature discrepancy).Then the heat conduction equation takes the form

$$
\left(K \varphi_{, i}\right)_{, i}=\left(1+\tau_{0} \frac{\partial}{\partial t}\right)\left(\rho C_{E} \frac{\partial \theta}{\partial t}+\gamma T_{0} \frac{\partial e}{\partial t}-Q\right)
$$

\section{Statement of the problem}

Let us consider a homogeneous thermoelastic half-space with two-temperatures occupying the region $z \geqslant 0$. The $z$-axis is taken perpendicular to the bounding plane pointing inwards. It is assumed that the initial state of the medium is quiescent. The surface of the medium is subjected to an inclined load. The inclined load is assumed to be a linear combination of the normal and tangential load. A Cartesian coordinate system $(x, y, z)$ is used. The present study is restricted to plane strain parallel to the $x z$-plane. The problem is thus two-dimensional with all functions considered depending on the spatial variables $x$ and $z$ as well as on the time variable $t$. The problem is considered within the context of the theory of two temperature thermoelasticity.

The displacement vector $\mathbf{u}$, thus, has the form

$$
\mathbf{u}=[u, 0, w]
$$

For the 2-D problem in the $x z$-plane, Eq. (2.1) can be written as

$$
\begin{aligned}
& (\lambda+2 \mu) \frac{\partial^{2} u}{\partial x^{2}}+(\lambda+\mu) \frac{\partial^{2} w}{\partial x \partial z}+\mu \frac{\partial^{2} u}{\partial z^{2}}-\gamma \frac{\partial \theta}{\partial x}=\rho \frac{\partial^{2} u}{\partial t^{2}} \\
& (\lambda+2 \mu) \frac{\partial^{2} w}{\partial z^{2}}+(\lambda+\mu) \frac{\partial^{2} u}{\partial x \partial z}+\mu \frac{\partial^{2} w}{\partial x^{2}}-\gamma \frac{\partial \theta}{\partial z}=\rho \frac{\partial^{2} w}{\partial t^{2}}
\end{aligned}
$$

The generalized equation of heat conduction, Eq. (2.8), is written in the $x z$-plane as

$$
K\left(\frac{\partial^{2} \varphi}{\partial x^{2}}+\frac{\partial^{2} \varphi}{\partial z^{2}}\right)=\left(1+\tau_{0} \frac{\partial}{\partial t}\right)\left(\rho C_{E} \frac{\partial \theta}{\partial t}+\gamma T_{0} \frac{\partial e}{\partial t}\right)
$$

and, Eq. (2.7) becomes

$$
\theta-\varphi=-a\left(\frac{\partial^{2} \varphi}{\partial x^{2}}+\frac{\partial^{2} \varphi}{\partial z^{2}}\right)
$$

In addition, the constitutive relations may be reduced to

$$
\begin{aligned}
\sigma_{x x}=(\lambda+2 \mu) \frac{\partial u}{\partial x}+\lambda \frac{\partial w}{\partial z}-\gamma \theta & \sigma_{y y}=\lambda\left(\frac{\partial u}{\partial x}+\frac{\partial w}{\partial z}\right)-\gamma \theta \\
\sigma_{z z}=(\lambda+2 \mu) \frac{\partial w}{\partial z}+\lambda \frac{\partial u}{\partial x}-\gamma \theta & \sigma_{x z}=\mu\left(\frac{\partial u}{\partial z}+\frac{\partial w}{\partial x}\right)
\end{aligned}
$$




\section{Solution of the problem}

Let us define displacement potentials $\Phi$ and $\Psi$ which are related to the displacements $u$ and $w$ as

$$
u=\frac{\partial \Phi}{\partial x}-\frac{\partial \Psi}{\partial z} \quad w=\frac{\partial \Phi}{\partial z}+\frac{\partial \Psi}{\partial x}
$$

Using the above relations in the governing equations, one obtains

$$
\begin{aligned}
& c_{1}^{2} \nabla^{2} \Phi-\frac{\partial^{2} \Phi}{\partial t^{2}}=\frac{\gamma}{\rho}\left(\varphi-a \nabla^{2} \varphi\right) \quad c_{2}^{2} \nabla^{2} \Psi-\frac{\partial^{2} \Psi}{\partial t^{2}}=0 \\
& K \nabla^{2} \varphi=\left(1+\tau_{0} \frac{\partial}{\partial t}\right)\left(\rho C_{E} \frac{\partial}{\partial t}\left[\varphi-a \nabla^{2} \varphi\right]+\gamma T_{0} \frac{\partial}{\partial t}\left(\nabla^{2} \Phi\right)\right)
\end{aligned}
$$

and

$$
\begin{aligned}
\sigma_{x x}=\lambda \nabla^{2} \Phi+2 \mu \frac{\partial}{\partial x}\left(\frac{\partial \Phi}{\partial x}-\frac{\partial \Psi}{\partial z}\right)-\gamma\left[\varphi-a \nabla^{2} \varphi\right] & \sigma_{y y}=\lambda \nabla^{2} \Phi-\gamma\left[\varphi-a \nabla^{2} \varphi\right] \\
\sigma_{z z}=\lambda \nabla^{2} \Phi+2 \mu \frac{\partial}{\partial z}\left(\frac{\partial \Psi}{\partial x}+\frac{\partial \Phi}{\partial z}\right)-\gamma\left[\varphi-a \nabla^{2} \varphi\right] & \sigma_{x z}=2 \mu \frac{\partial^{2} \Phi}{\partial x \partial z}+\mu\left(\frac{\partial^{2} \Psi}{\partial x^{2}}-\frac{\partial^{2} \Psi}{\partial z^{2}}\right)
\end{aligned}
$$

where

$$
c_{1}^{2}=\frac{\lambda+2 \mu}{\rho} \quad c_{2}^{2}=\frac{\mu}{\rho} \quad \nabla^{2}=\frac{\partial^{2}}{\partial x^{2}}+\frac{\partial^{2}}{\partial z^{2}}
$$

The initial conditions are given by

$$
\begin{array}{ll}
u(x, z, 0)=w(x, z, 0)=0 & \frac{\partial u}{\partial t}=\frac{\partial w}{\partial t}=0 \\
\theta(x, z, 0)=\varphi(x, z, 0)=0 & \frac{\partial \varphi}{\partial t}=\frac{\partial \theta}{\partial t}=0
\end{array}
$$

The following dimensionless variables are introduced

$$
\begin{array}{lll}
\left\{x^{\prime}, z^{\prime}, u^{\prime}, w^{\prime}\right\}=\frac{\eta_{0}}{c_{1}}\{x, z, u, w\} & \left\{t^{\prime}, \tau_{0}^{\prime}\right\}=\eta_{0}\left\{t, \tau_{0}\right\} & \eta_{0}=\frac{\rho C_{E} c_{1}^{2}}{K} \\
\left\{\Phi^{\prime}, \Psi^{\prime}, a^{\prime}\right\}=\left(\frac{\eta_{0}}{c_{1}}\right)^{2}\{\Phi, \Psi, a\} & \left\{\theta^{\prime}, \varphi^{\prime}\right\}=\frac{\gamma}{\rho c_{1}^{2}}\{\theta, \varphi\} & \sigma_{i j}^{\prime}=\frac{\sigma_{i j}}{\mu}
\end{array}
$$

into Eqs. (4.2). After removing the primes, one obtains

$$
\begin{aligned}
& \nabla^{2} \Phi-\frac{\partial^{2} \Phi}{\partial t^{2}}=\varphi-a \nabla^{2} \varphi \quad \nabla^{2} \Psi-\beta^{2} \frac{\partial^{2} \Psi}{\partial t^{2}}=0 \\
& {\left[1+\eta_{0} a \frac{\partial}{\partial t}\left(1+\tau_{0} \frac{\partial}{\partial t}\right)\right] \nabla^{2} \varphi=\eta_{0}\left(1+\tau_{0} \frac{\partial}{\partial t}\right)\left(\frac{\partial \varphi}{\partial t}+\varepsilon \frac{\partial}{\partial t}\left(\nabla^{2} \Phi\right)\right)}
\end{aligned}
$$

where $\varepsilon$ is the dimensionless thermoelastic coupling constant and $\beta$ is the ratio of the longitudinal waves speed to the shear waves speed

$$
\varepsilon=\frac{\gamma^{2} T_{0}}{\rho C_{E}(\lambda+2 \mu)} \quad \beta^{2}=\frac{\lambda+2 \mu}{\mu}
$$

Here, we restrict our attention to the constitutive relations given by Eqs. (4.3) $)_{3,4}$. They become

$$
\begin{aligned}
\sigma_{z z} & =2 \frac{\partial}{\partial z}\left(\frac{\partial \Psi}{\partial x}+\frac{\partial \Phi}{\partial z}\right)+\left(1-2 \beta^{2}\right) \nabla^{2} \Phi-\beta^{2}\left[\varphi-a \nabla^{2} \varphi\right] \\
\sigma_{x z} & =2 \frac{\partial^{2} \Phi}{\partial x \partial z}+\frac{\partial^{2} \Psi}{\partial x^{2}}-\frac{\partial^{2} \Psi}{\partial z^{2}}
\end{aligned}
$$




\section{Normal mode analysis}

The normal mode analysis is used to give exact solutions without any assumed restrictions on temperature, displacement, and stress distributions (Cheng and Zhang, 2000; Allam et al., 2009). The solution of the present field quantities can be decomposed in terms of the normal modes as

$$
\left[u, w, \varphi, \Phi, \Psi, \sigma_{i j}\right](x, y, t)=\left[u^{*}, w^{*}, \varphi^{*}, \Phi^{*}, \Psi^{*}, \sigma_{i j}^{*}\right](x) \mathrm{e}^{(\omega t+\mathrm{i} \zeta z)}
$$

where $\omega$ is the complex frequency, $\mathrm{i}=\sqrt{-1}, \zeta$ is the wave number in the $z$-direction, and $u^{*}(x)$, $w^{*}(x), \varphi^{*}(x), \Phi^{*}(x), \Psi^{*}(x)$ and $\sigma_{i j}^{*}(x)$ are the amplitudes of the field quantities. Using Eq. (34), Eqs. (4.7) take the forms

$$
\left(D^{2}-\alpha_{1}\right) \Phi^{*}=-a\left(D^{2}-\alpha_{2}\right) \varphi^{*} \quad q \varepsilon\left(D^{2}-\zeta^{2}\right) \Phi^{*}=\left(D^{2}-\alpha_{3}\right) \varphi^{*} \quad\left(D^{2}-k_{3}^{2}\right) \Psi^{*}=0
$$

where

$$
\begin{aligned}
& \alpha_{1}=\zeta^{2}+\omega^{2} \quad \alpha_{2}=\zeta^{2}+\frac{1}{a} \quad \alpha_{3}=\zeta^{2}+q \quad k_{3}^{2}=\zeta^{2}+\omega^{2} \beta^{2} \\
& q=\frac{\omega \eta_{0}\left(1+\tau_{0} \omega\right)}{1+\eta_{0} a \omega\left(1+\tau_{0} \omega\right)} \quad D=\frac{\mathrm{d}}{\mathrm{dx}}
\end{aligned}
$$

Eliminating $\varphi^{*}(x)$ or $\Phi^{*}(x)$ in Eqs. (5.2) $)_{1,2}$, one gets the following fourth-order differential equation for $\varphi^{*}(x)$ or $\Phi^{*}(x)$

$$
\left(D^{4}-A D^{2}+B\right)\left\{\varphi^{*}(x), \Phi^{*}(x)\right\}=0
$$

where

$$
A=\frac{\alpha_{1}+\alpha_{3}-q \varepsilon a\left(\zeta^{2}+\alpha_{2}\right)}{1-q \varepsilon a} \quad B=\frac{\alpha_{1} \alpha_{3}-q \varepsilon a \zeta^{2} \alpha_{2}}{1-q \varepsilon a}
$$

Introducing $k_{i}(i=1,2,3)$ into Eq. (5.4), one finds

$$
\left(D^{2}-k_{1}^{2}\right)\left(D^{2}-k_{2}^{2}\right)\left\{\varphi^{*}(x), \Phi^{*}(x)\right\}=0
$$

where $k_{1}^{2}$ and $k_{2}^{2}$ are the roots of the characteristic equation

$$
k^{4}-A k^{2}+B=0
$$

The roots of Eqs. (5.6) are given by

$$
k_{1}^{2}=\frac{1}{2}\left[2 A+\sqrt{A^{2}-4 B}\right] \quad k_{2}^{2}=\frac{1}{2}\left[2 A+\sqrt{A^{2}-4 B}\right]
$$

Keeping in mind that $\varphi^{*}, \Phi^{*} \rightarrow 0$ as $x \rightarrow \infty$ for surface waves, the solution to Eq. (5.6) has the form

$$
\left\{\varphi^{*}(x), \Phi^{*}(x)\right\}=\sum_{n=1}^{2}\left\{m_{n}(a, \omega), M_{n}(a, \omega)\right\} \mathrm{e}^{-k_{n} x}
$$

where $m_{n}(a, \omega)$ and $M_{n}(a, \omega)$ are specific functions depending on $a$ and $\omega$. Substituting Eqs. (5.9) into Eqs. (5.2) $)_{2}$, one obtains the following relation

$$
M_{n}(a, \omega)=H_{n} m_{n}(a, \omega) \quad n=1,2
$$


where

$$
H_{n}=\frac{k_{n}^{2}-\alpha_{3}}{q \varepsilon\left(k_{n}^{2}-\zeta^{2}\right)} \quad n=1,2
$$

Thus, one has

$$
\left\{\varphi^{*}(x), \Phi^{*}(x)\right\}=\sum_{n=1}^{2}\left\{1, H_{n}\right\} m_{n}(a, \omega) \mathrm{e}^{-k_{n} x}
$$

From the regularity condition, one obtains

$$
\Psi^{*}(x)=m_{3}(a, \omega) \mathrm{e}^{-k_{3} x}
$$

where $m_{3}(a, \omega)$ is specific function depending on $a$ and $\omega$. Making use of solutions given in Eqs. (5.12) and (5.13), one gets

$$
u^{*}=-\sum_{n=1}^{2} k_{n} H_{n} m_{n} \mathrm{e}^{-k_{n} x}-\mathrm{i} \zeta m_{3} \mathrm{e}^{-k_{3} x} \quad w^{*}=\mathrm{i} \zeta \sum_{n=1}^{2} H_{n} m_{n} \mathrm{e}^{-k_{n} x}-k_{3} m_{3} \mathrm{e}^{-k_{3} x}
$$

Using Eqs. (3.4) and (5.9), one obtains

$$
\theta^{*}=\sum_{n=1}^{2}\left[\left(1+a \zeta^{2}\right)-a k_{n}^{2}\right] m_{n} \mathrm{e}^{-k_{n} x}
$$

Substituting Eqs. (5.12) and (5.13) into Eqs. (4.8), we obtain

$$
\begin{aligned}
& \sigma_{z z}^{*}=\sum_{n=1}^{2} L_{n} m_{n} \mathrm{e}^{-k_{n} x}-2 \mathrm{i} \zeta k_{3} m_{3} \mathrm{e}^{-k_{3} x} \\
& \sigma_{x z}^{*}=-2 \mathrm{i} \zeta \sum_{n=1}^{2} k_{n} H_{n} m_{n} \mathrm{e}^{-k_{n} x}+\left(\zeta^{2}+k_{3}^{2}\right) m_{3} \mathrm{e}^{-k_{3} x}
\end{aligned}
$$

where

$$
L_{n}=\left[-2 \zeta^{2}+\left(1-2 \beta^{2}\right)\left(k_{n}^{2}-\zeta^{2}\right)\right] H_{n}-\beta^{2}\left[1-a\left(k_{n}^{2}-\zeta^{2}\right)\right]
$$

\section{Applications}

In what follows, the parameters $m_{j}(j=1,2,3)$ will be determined. In a physical problem, we should suppress the positive exponentials that are unbounded at infinity. Suppose that an inclined line load $F_{0}$ is acting along the interface on the $x$-axis and its inclination angle with the $z$-axis is $\theta_{0}$.

The normal line load $F_{1}$ is considered to be acting in the positive $x$ direction on the surface $x=0$ along the $z$-axis, and the tangential load $F_{2}$ is acting at the origin in the positive $x$ direction, then the boundary conditions on the surface $y=0$ are

$$
\left\{\sigma_{z z}(0, z, t), \sigma_{x y}(0, z, t)\right\}=-\left\{F_{1}, F_{2}\right\} \mathrm{e}^{(\omega t+\mathrm{i} \zeta z)}
$$

where for the inclined line load $F_{0}$ we have $F_{1}=F_{0} \cos \theta_{0}$ and $F_{2}=F_{0} \sin \theta_{0}$.

The surface $x=0$ is thermally insulated, i.e. it satisfies the boundary condition

$$
\frac{\partial \varphi(0, z, t)}{\partial x}=0
$$


Using the above boundary conditions, one readily obtains the following equations

$$
\begin{aligned}
& \sum_{n=1}^{2} L_{n} m_{n}-2 \mathrm{i} \zeta k_{3} m_{3}=-F_{1} \quad-2 \mathrm{i} \zeta \sum_{n=1}^{2} k_{n} H_{n} m_{n}+\left(\zeta^{2}+k_{3}^{2}\right) m_{3}=-F_{2} \\
& \sum_{n=1}^{2} k_{n} m_{n}(a, \omega)=0
\end{aligned}
$$

They may be written in the matrix form

$$
\left\{\begin{array}{l}
m_{1} \\
m_{2} \\
m_{3}
\end{array}\right\}=\left[\begin{array}{ccc}
L_{1} & L_{2} & -2 \mathrm{i} \zeta k_{3} \\
-2 \mathrm{i} \zeta k_{1} H_{1} & -2 \mathrm{i} \zeta k_{2} H_{2} & \zeta^{2}+k_{3}^{2} \\
k_{1} & k_{2} & 0
\end{array}\right]^{-1}\left\{\begin{array}{c}
-F_{1} \\
-F_{2} \\
0
\end{array}\right\}
$$

which gives values of the three constants $m_{j}(j=1,2,3)$. Therefore, the physical quantities of the medium will be fully determined.

\section{Numerical results}

Numerical results of the thermodynamical temperature, conductive temperature, displacements and stresseswill be presented here. In the present work, the thermoelastic solid half-space due to an inclined load is analyzed by considering the medium made of Copper. The physical properties of Copper is presented in Table 1.

Table 1. Physical data of Copper

\begin{tabular}{|c|c|c|}
\hline Quantity & Unit & Value \\
\hline \hline$K$ & $\mathrm{~W} /(\mathrm{m} \mathrm{K})$ & 368 \\
\hline$\rho$ & $\mathrm{kg} / \mathrm{m}^{3}$ & 8954 \\
\hline$C_{E}$ & $\mathrm{~J} /(\mathrm{kg} \mathrm{K})$ & 383.1 \\
\hline$\alpha_{t}$ & $1 / \mathrm{K}$ & $1.78 \cdot 10^{-5}$ \\
\hline$\lambda$ & $\mathrm{N} / \mathrm{m}^{2}$ & $7.76 \cdot 10^{10}$ \\
\hline$\mu$ & $\mathrm{N} / \mathrm{m}^{2}$ & $3.86 \cdot 10^{10}$ \\
\hline$T_{0}$ & $\mathrm{~K}$ & 293 \\
\hline
\end{tabular}

The computations are carried out on the surface $z=1.0$ at $t=0.15$. The graphical results for the dimensionless temperature distribution $\theta$, conductive temperature $\varphi$, normal displacement $u$, transverse displacement $w$, normal stress $\sigma_{z z}$, and tangential stress $\sigma_{z x}$ are shown in Figs. 1 and 2 with $F_{0}=1, \omega=\omega_{0}+\mathrm{i} \xi, \omega_{0}=2, \xi=0.1, \zeta=2.1$. All the functions have been evaluated inside the medium on the $z$-axis $(z=1.0)$ as functions of $x$.

A number of special cases are pertinent in this contribution:

- The equations of the coupled thermoelasticity (CTE) theory with one temperature are obtained when $\tau_{0}=0$ and $a \rightarrow 0$.

- The equations of the coupled thermoelasticity (CTE theory) with two temperatures are obtained when $\tau_{0}=0$ and $a>0$.

- The equations of the Lord-Shulman (LS) theory with one relaxation time are retrieved when $\tau_{0}>0$ and $a \rightarrow 0$.

- The equations of the generalized thermoelasticity with two temperatures are obtained when $\tau_{0}>0$ and $a>0$. 
(a)

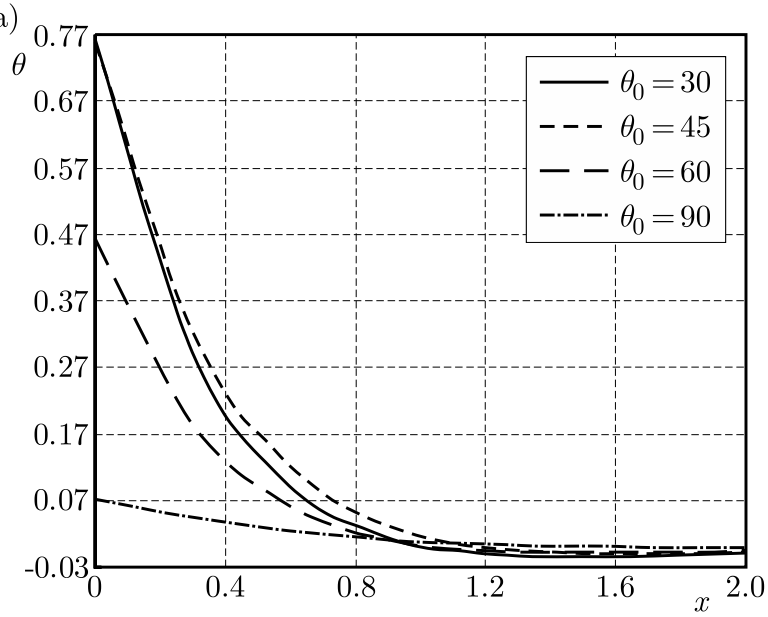

(b)

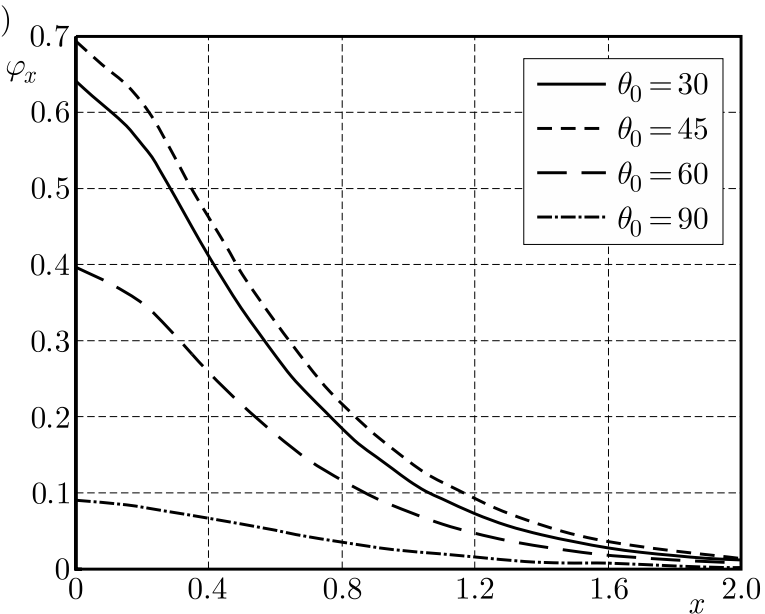

(c)

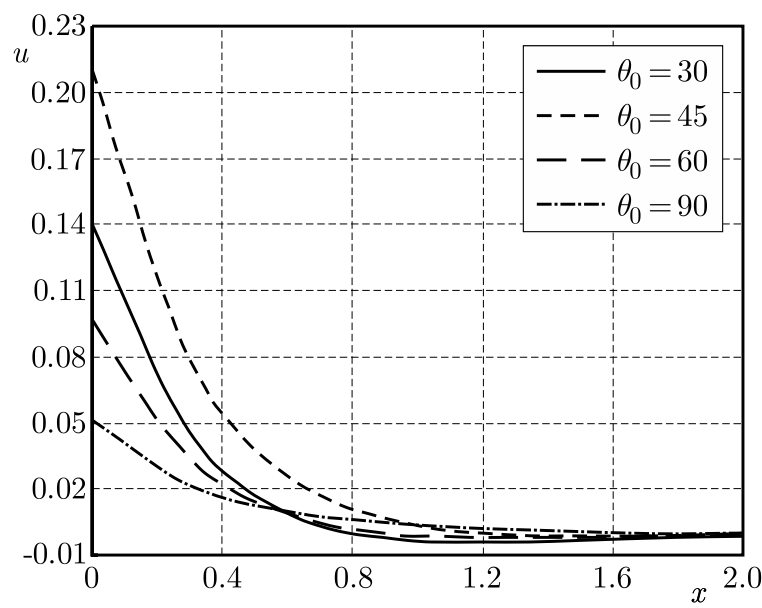

(d)

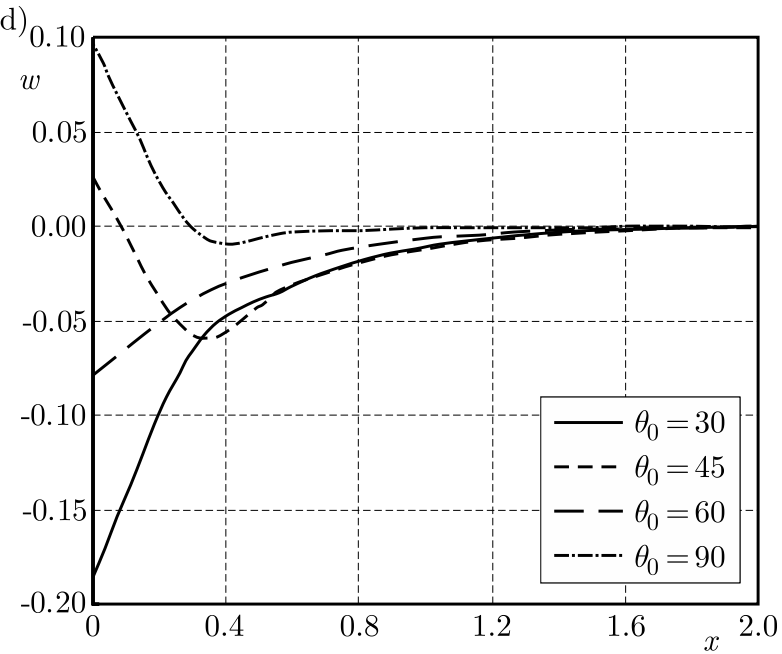

(e)

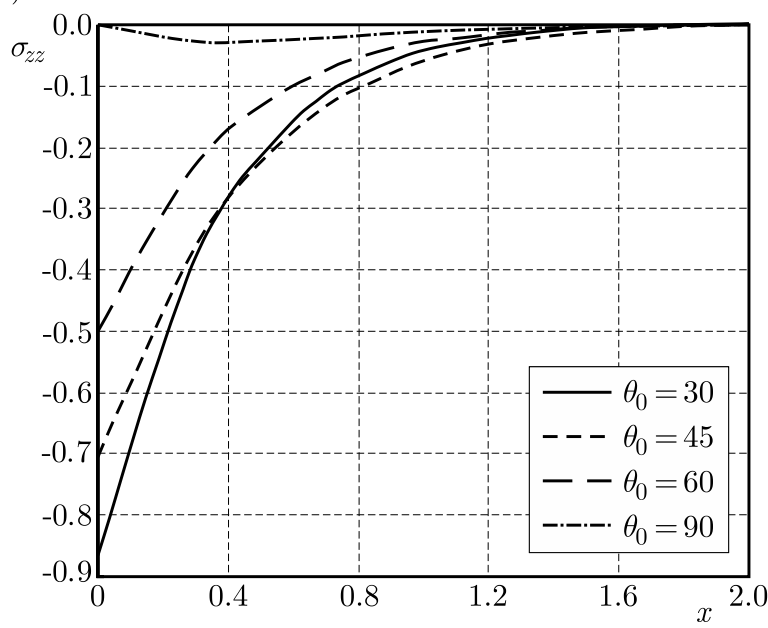

(f)

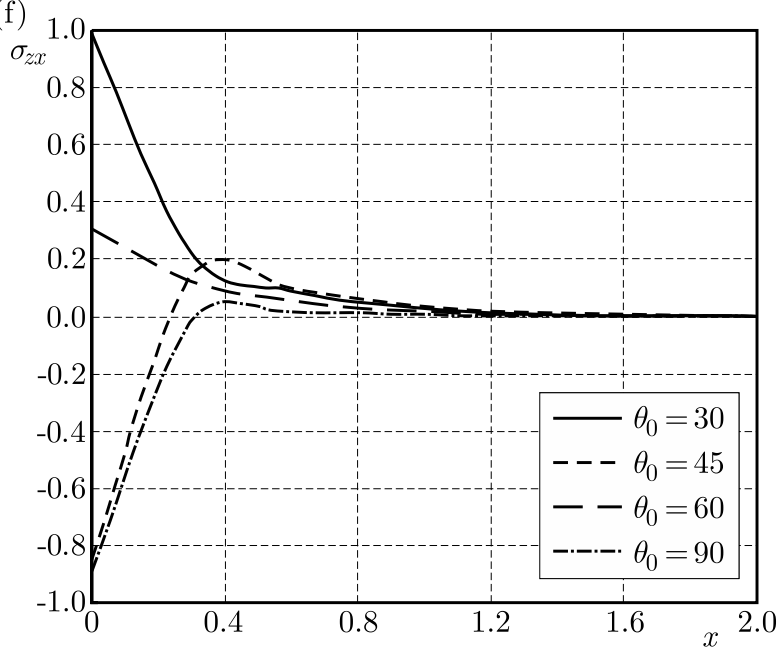

Fig. 1. Variation of dimensionless (a) temperature distribution $\theta$, (b) conductive $\varphi$,

(c) normal displacement $u$, (d) tangential displacement $w$, (e) normal force stress $\sigma_{z z}$,

(f) tangential force stress $\sigma_{z x}$ with distance $x$ for different values of angle of inclination $\theta_{0}$

Figures 1a-1f give a comparison of the results obtained for the normal displacement $u$, tangential displacement $v$, transverse normal stress $\sigma_{z z}$, tangential shear stress $\sigma_{z x}$, temperature distribution $\theta$ and conductive temperature $\varphi$ against the $x$ direction for different values of the angle of inclination $\theta_{0}$ (orientation of source) and the temperature discrepancy parameter remains constant. 
We can see that the angle of inclination $\theta_{0}$ has significant effects on all the studied fields. It has been observed that $\theta_{0}$ plays a vital role on the development of temperature, stress, and displacement fields, in which the following is observed:

1. A significant difference in values of the studied fields is noticed for different values of the angle of inclination $\theta_{0}$.

2. Values of temperature, conductive temperature and normal displacement in the fixed point $(x, z)$ increase when $0 \leqslant \theta \leqslant \pi / 4$ and decrease when $\pi / 3 \leqslant \theta \leqslant \pi / 2$ for the whole time $t$.

3. The maximum points of displacements $w$ increase when $\theta_{0}$ increases.

4. Figure $1 \mathrm{~d}$ shows that the behavior due to $\theta_{0}=45^{\circ}$ and $\theta_{0}=90^{\circ}$ is similar, and so for $\theta_{0}=30^{\circ}$ and $\theta_{0}=60^{\circ}$.

5. The angle of inclination $\theta_{0}$ has large effects on the value of the tangential shear stress $\sigma_{z x}$ which means that $\sigma_{z x}$ decreases with $\theta_{0}$.

6. Values of the normal stress $\sigma_{z z}$ in the fixed point $(x, z)$ decrease when $0 \leqslant \theta \leqslant \pi / 4$ and increase when $\pi / 3 \leqslant \theta \leqslant \pi / 2$ at any moment $t$.

Figures $2 \mathrm{a}-2 \mathrm{f}$ show how the field results vary with different values of the dimensionless temperature discrepancy. The case $a \rightarrow 0$ indicates the old situation (one temperature 1TT) and the cases $a>0$ indicate the two-temperature theory (2TT).

The variation is very sensitive to the response of all field quantities. When $a=0$, all quantities exhibit different behavior. This shows the difference between the one temperature generalized thermoelasticity of LS (1TT) and the two-temperature generalized thermoelasticity (2TT). Also, Figs. 2a-2f show that this parameter has significant effects on all the field quantities. The waves reach the steady state depending on the value of the temperature discrepancy. It can be observed that $a>0$ corresponds to a slower rate of decay than the case when $a=0$. Once again, the behavior of 2TT model may differ from that of 1TT model near the boundary plane. The model of thermoelasticity with two temperatures predicts a finite speed of wave propagation, which makes the generalized theorem of thermoelasticity more agreeable with physical properties of the material.

\section{Conclusions}

Analysis of normal displacement, tangential displacement, transverse normal stress, tangential shear stress, temperature distribution and conductive temperature due to mechanical load in a semi-infinite generalized thermoelastic medium is an interesting problem of mechanics. The generalized two-temperature theory of thermoelasticity in the context of Lord and Shulman's (LS) model is used to solve this problem. A normal mode technique has been used which is applicable to a wide range of problems of thermoelasticity. This method gives exact solutions without any assumed restrictions on the actual physical quantities that appear in the governing equations of the problem considered. The effects of the angle of inclination as well as the twotemperature parameter on the field variables are investigated. The results concluded from the above analysis can be summarized as follows:

- It is seen that the values of all the field variables are significantly dependent on the twotemperature parameter.

- According to the theory of thermoelasticity with two temperatures, we have to construct a new classification for materials according to their fractional parameter. This parameter becomes a new indicator of the material ability to conduct heat under the effect of thermoelastic properties. 
(a)

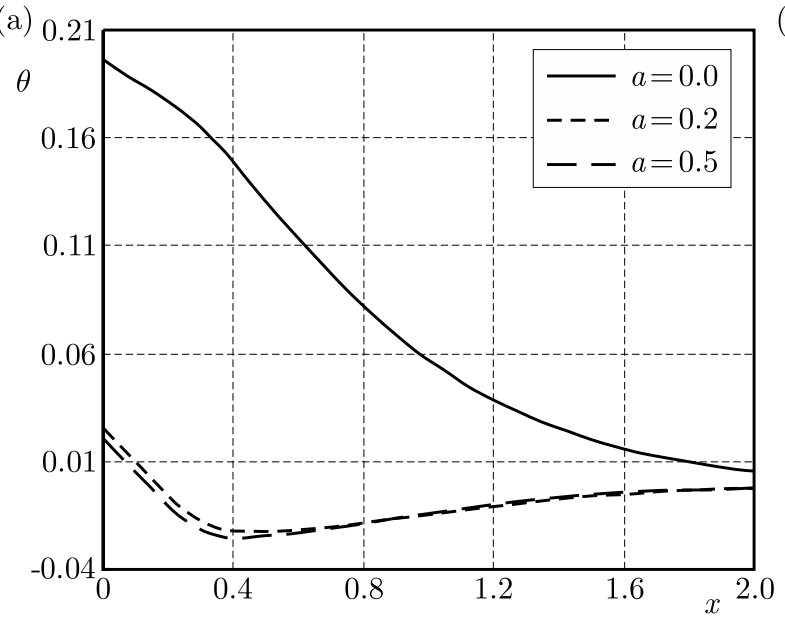

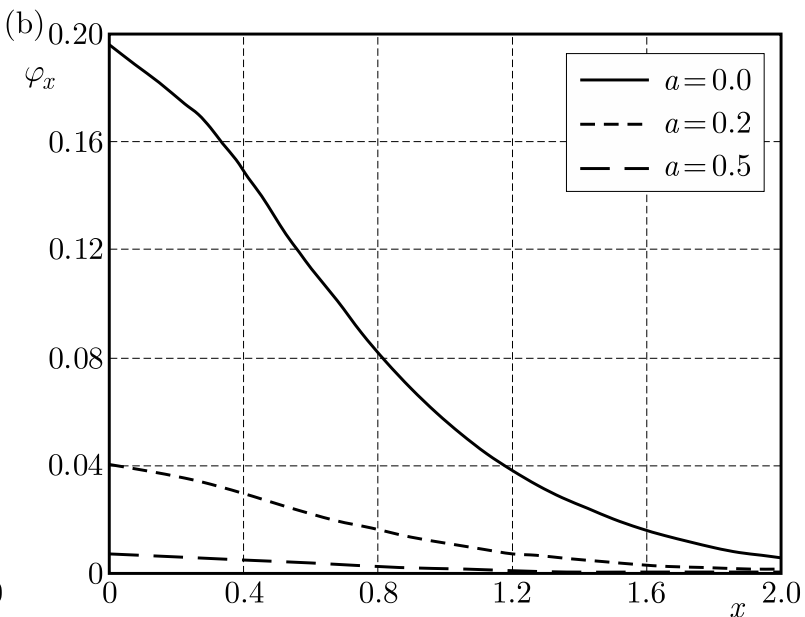

(d)

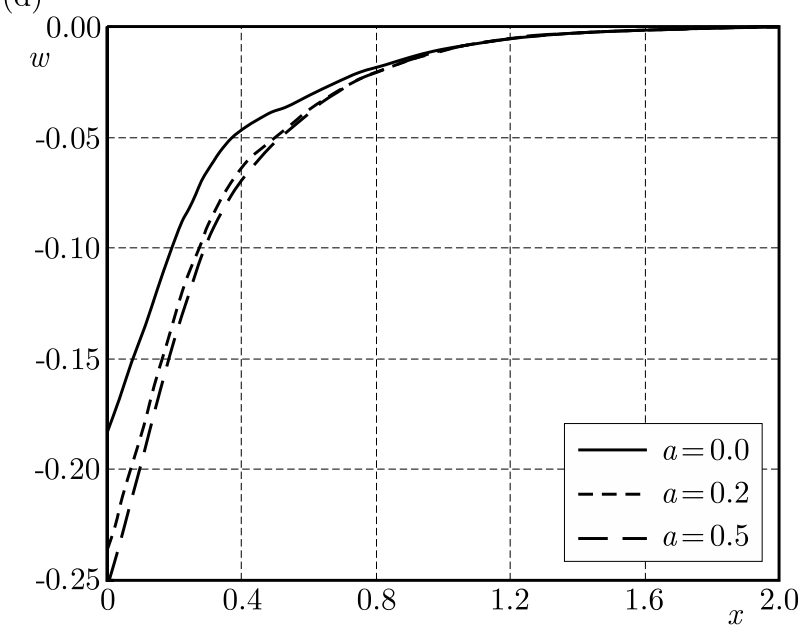

(c)

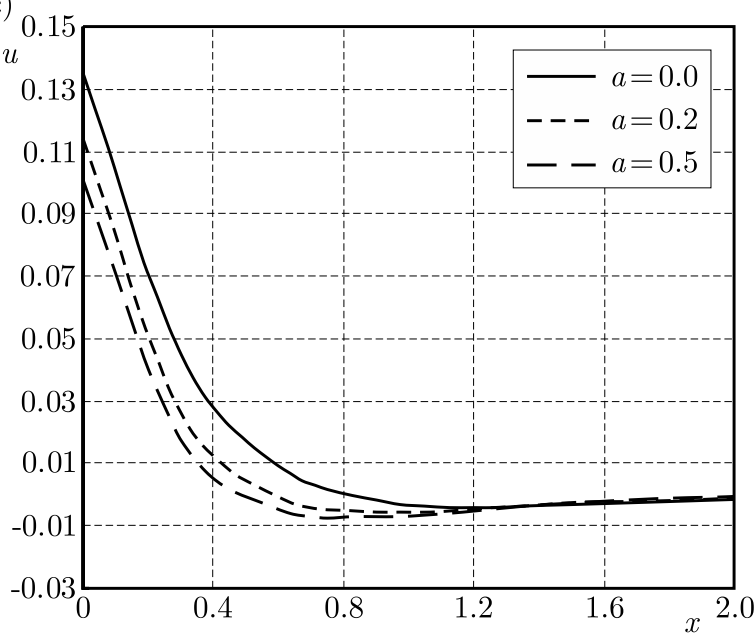

(f)

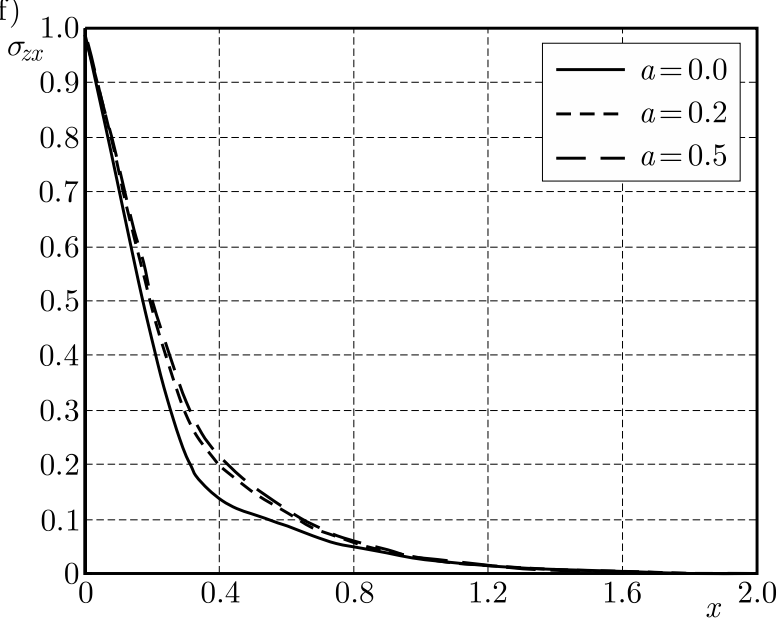

(e)

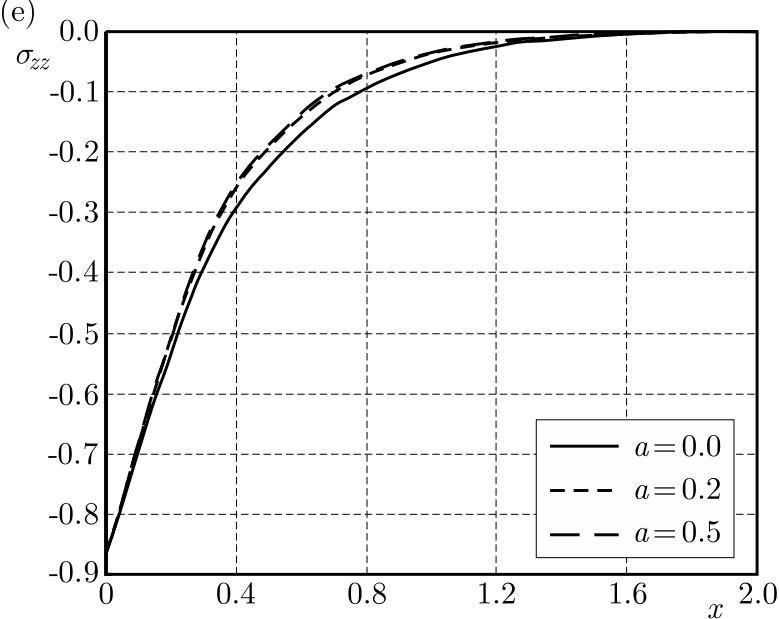

Fig. 2. Variation of dimensionless (a) temperature distribution $\theta$, (b) conductive $\varphi$,

(c) normal displacement $u$, (d) tangential displacement $w$, (e) normal force stress $\sigma_{z z}$,

(f) tangential stress $\sigma_{z x}$ with distance $x$ for different values of temperature discrepancy parameter $a$

- It is also observed that the theories of coupled thermoelasticity and generalized thermoelasticity with one relaxation time can be obtained as limit cases.

- According to this work, one can consider the theory of two-temperature generalized thermoelasticity as an improvement of the study on elastic materials. 
- The properties of the body largely depend on the direction of symmetry and the inclination of the applied source.

- Significant difference in values of the studied fields is noticed for different values of the angle of inclination.

\section{References}

1. Abbas I.A., Zenkour A.M., 2014, Two-temperature generalized thermoelastic interaction in an infinite fiber-reinforced anisotropic plate containing a circular cavity with two relaxation times, Journal of Computational and Theoretical Nanoscience, 11, 1, 1-7

2. Abouelregal A.E., Zenkour A.M., 2013, Effect of fractional thermoelasticity on a twodimensional problem of a mode I crack in a rotating fibre-reinforced thermoelastic medium, Chinese Physics B, 22, 10, 108102

3. Allam M.N., Elsibai K.A., Abouelregal A.E., 2009, Electromagneto-thermoelastic problem in a thick plate using Green and Naghdi theory, International Journal of Engineering Science, 47, $680-690$

4. Biot M.A., 1956, Thermoelasticity and irreversible thermodynamics, Journal of Applied Physics, 27, 240-253

5. Boley M., 1956, Thermoelastic and irreversible thermo dynamics, Journal of Applied Physics, 27, $240-253$

6. Chen P.J., Gurtin M.E., 1968, On a theory of heat conduction involving two temperatures, Zeitschrift für angewandte Mathematik und Physik ZAMP, 19, 614-627

7. Chen P.J., Gurtin M.E., Willams W.O., 1969, On the thermodynamics of non-simple elastic material with two temperatures, Zeitschrift für angewandte Mathematik und Physik ZAMP, 20, $107-112$

8. Cheng J.C., Zhang S.Y., 2000, Normal mode expansion method for laser generated ultrasonic lamb waves in orthotropic thin plates, Applied Physics B, 70, 57-63

9. El-Maghraby N.M., 2008, A Two-dimensional generalized thermoelasticity problem for a halfspace under the action of a body force, Journal of Thermal Stresses, 31, 557-568

10. El-Maghraby N.M., 2009, Two-dimensional thermoelasticity problem for a thick plate under the action of a body force in two relaxation times, Journal of Thermal Stresses, 32, 863-876

11. Green A.E., Lindsay K.A., 1972, Thermoelasticity, Journal of Elasticity, 2, 1-7

12. Green A.E. NAGhdi P.M., 1993, Thermoelasticity without energy dissipation, Journal of Elasticity, 31, 189-208

13. Lord H.W., Shulman Y., 1967, A generalized dynamical theory of thermoelasticity, Journal of the Mechanics and Physics of Solids, 15, 299-309

14. Sherief H., Hamza F., 1996, Generalized two dimensional thermoelastic problems in spherical regions under axisymmetric distributions, Journal of Thermal Stresses, 19, 55-76

15. Sherief H.H., El-Maghraby N., 2003, An internal penny-shaped crack in an infinite thermoelastic solid, Journal of Thermal Stresses, 26, 333-352

16. Sherief H.H., El-Maghraby N., 2005, A mode-I crack in an Infinite Space in Generalized Thermoelasticity, Journal of Thermal Stresses, 28, 465-484

17. Zenkour A.M., 2015, Three-dimensional thermal shock plate problem within the framework of different thermoelasticity theories, Composite Structures, 132, 1029-1042

18. Zenkour A.M., Abouelregal A.E., 2014a, State-space approach for an infinite medium with a spherical cavity based upon two-temperature generalized thermoelasticity theory and fractional heat conduction, Zeitschrift für angewandte Mathematik und Physik ZAMP, 65, 1, 149-164 
19. Zenkour A.M., Abouelregal A.E., 2014b, The effect of two temperatures on a functionally graded nanobeam induced by a sinusoidal pulse heating, Structural Engineering and Mechanics, 51, 2, 199-214

20. Zenkour A.M., Abouelregal A.E., 2015, Thermoelastic problem of an axially moving microbeam subjected to an external transverse excitation, Journal of Theoretical and Applied Mechanics, 53, $167-178$

Manuscript received December 25, 2014; accepted for print November 29, 2015 